\title{
EFEITO DO NITROGÊNIO E POTÁSSIO NA NUTRIÇÃO DO PIMENTÃO CULTIVADO EM AMBIENTE PROTEGIDO(1)
}

\author{
M. A. G. SILVA(2), A. E. BOARETTO(3), T. MURAOKA(3), \\ H. G. FERNANDE $S^{(4)}$, F. A. GRANJ A(5) \& W. B. SCIVITTARO ${ }^{(6)}$
}

\begin{abstract}
RESUMO
Avaliou-se a influência do $\mathbf{N}$ e $\mathrm{K}$ na concentração e acúmulo de nutrientes no pimentão, cultivar Mayata, irrigado por gotejamento. O experimento foi instalado em cultivo protegido telado, em Latossolo Vermel ho mesoférrico, entre - período de novembro de 1996 a agosto de 1997, durante 34 semanas. Os tratamentos constaram de três doses de $\mathrm{N}\left(13,3 ; 26,6\right.$ e 39,9 $\left.\mathrm{g} \mathrm{m}^{-2}\right)$, como uréia, e três doses de $\mathrm{K}\left(5,5 ; 11,0\right.$ e 16,6 $\left.\mathrm{g} \mathrm{m}^{-2}\right)$, como $\mathrm{KCl}$, distribuídas em esquema fatorial, utilizando o delineamento em blocos casualizados. Todas as plantas foram irrigadas por gotejamento tendo sido a uréia e o cloreto de potássio aplicados logo abaixo dos gotejadores. As doses de $\mathbf{N}$ não afetaram a concentração dos nutrientes em folhas recém-maduras, no início do florescimento e início da frutificação, enquanto o $\mathrm{K}$ diminuiu a concentração de $P$ e $S$, no início da frutificação, exceto na dose de $5,5 \mathrm{~g} \mathrm{~m}^{-2}$ de $\mathrm{K}$. $\mathrm{O} \mathrm{N}$ aumentou o acúmulo de nutrientes na parte aérea, ao final do ciclo de cultivo, quando aplicado na dose de $26,6 \mathrm{~g} \mathrm{~m}^{-2}$, porém não influenciou a produção de frutos. $0 \mathrm{~K}$ contribuiu para a absorção de nutrientes e produção de frutos, somente quando presente em baixas concentrações no solo. Doses de $K$ de $16,6 \mathrm{~g} \mathrm{~m}^{-2}$, relacionaram-se com a alta condutividade elétrica $\left(1.400 \mu \mathrm{S} \mathrm{cm}^{-1}\right)$, até a camada de $40 \mathrm{~cm}$, e com a alta concentração de cloreto na parte aérea e frutos de pimentão.
\end{abstract}

Termos de indexação: Capsicum annuum, adubação nitrogenada, adubação potássica, salinidade do solo.

(1) Pesquisa financiada pela Fundação de Amparo à Pesquisa do Estado de São Paulo - FAPESP. Recebido para publicação em fevereiro de 2000 e aprovado em junho de 2001.

(2) Professora do Departamento de Agronomia da Universidade Estadual de Maringá - UEM. Av. Colombo 5790, CEP 87020-900 Maringá (PR). E-mail: magsilva@uem.br

(3) Pesquisador do Centro de Energia Nuclear na Agricultura - CENA. Caixa Postal 96, CEP 13400-970 Piracicaba (SP). E-mail: aeboaret@cena.usp.br

(4) Bióloga do CENA. E-mail: hgimenes@cena.usp.br

(5) Estagiário do CENA.

(6) Pesquisadora da Embrapa Centro de Pesquisa de Clima Temperado. Br 392, Km 78, Caixa Postal 403, CEP $96001-970$ Pelotas (RS). E-mail: wbscivt@cpact.embrapa.br 


\title{
SUMMARY: NITROGEN AND POTASSIUM EFFECT ON THE NUTRITION OF PEPPER GROWN IN A PROTECTED ENVIRONMENT
}

\begin{abstract}
Nitrogen and potassium applications affected the nutrient concentration and accumulation of sweet pepper, cultivar Mayata, grown in a protected environment. An experiment was conducted in a protected environment in Eutrostox, during 34 weeks from November, 1996 to August, 1997, with the fol lowing treatments: control and combination of threerates of $\mathrm{N}\left(13.3 ; 26.6\right.$ and $\left.39.9 \mathrm{~g} \mathrm{~m}^{-2}\right)$ and thre rates of $\mathrm{K}\left(5.5 ; 11.0\right.$ and $\left.16.6 \mathrm{~g} \mathrm{~m}^{-2}\right)$. All plants were watered by trickle irrigation and the urea and $\mathrm{KCl}$ were applied below each trickler. Theresults led to thefollowing conclusions: $\mathrm{N}$ did not affect theconcentration of nutrients in recently-ripened leaves, in the beginning of flowering and fruiting, although $\mathrm{K}$, in high rates, decreased the concentration of $\mathrm{P}$ and $\mathrm{S}$, in the beginning of fruiting; $\mathrm{N}$ increased theabsorption of nutrients on the top part at the end of the cultivation period at the rate of $26.6 \mathrm{~g} \mathrm{~m}^{-2}$, but did not influence fruit production. Potassium contributed to absorption of nutrient and fruit production, only when concentrations in the soil were low. Patassium, in high rates $\left(16.6 \mathrm{~g} \mathrm{~m}^{-2}\right)$, related with high electric conductivity $\left(1.400 \mu \mathrm{S} \mathrm{cm}^{-1}\right)$, in a layer of $40 \mathrm{~cm}$ and high $\mathrm{Cl}$ concentration in the shoot and fruits.
\end{abstract}

Index terms: Capsicum annuum, nitrogen fertilization, potassium fertilization, soil sali nity.

\section{NTRODUÇÃO}

O pimentão (Capsi cum annuum L.), pertencente a família Solanaceae e ao gênero Capsicum, é originário da América Central, em regiões de clima tropical. Deacordo com Siviero \& Gallerani (1992), a faixa ideal de temperatura está entre mínima de $1^{\circ} \mathrm{C}$ e máxima de $30^{\circ} \mathrm{C}$.

O pimentão cultivado em ambiente protegido apresenta ciclo maior (em torno de 12 meses) e condições de maior crescimento, considerando as características favoráveis de cultivo; por isso, supõese que seja mais exigente do ponto de vista nutricional, em comparação ao cultivo em campo.

São poucos os trabalhos com adubação em condições de cultivo protegido. Nesse sentido, Valenzuela \& Romero (1996) estudaram a influência do N (180 kg ha-1) e do K (40 kg ha-1), aplicados com fertiirrigação na produção e nas exigências nutricionais do pimentão, e observaram diminuição no número, peso e rendimento dos frutos, para essas doses aplicadas aosol o. A dose de $\mathrm{N}$ quese relacionou com o maior rendimento de frutos de pimentão foi de $140 \mathrm{~kg} \mathrm{ha}^{-1}$, de acordo com Morales Payan et al. (1998) também em cultivo protegido. Moura \& Casali (1999) estudaram o efeito do P no comportamento do pimentão. A dose de $250 \mathrm{mg} \mathrm{kg}^{-1}$ de P no solo foi considerada a melhor para a produção de matéria seca da parte aérea, característica adequada para avaliar a eficiência do $P$ em estudos genéticos.

Em condi ções de campo, doses deaté $224 \mathrm{~kg} \mathrm{ha}^{-1}$ de $\mathrm{N}$ estiveram relacionadas com a alta produção de matéria seca e produtividade do pimentão (Locascio et al., 1985), assim como doses de K (180 kg ha-1) estimularam o crescimento e a produção do pimentão, de acordo com Gollifer (1993).

Na prática, a aplicação excessiva de fertilizantes pode levar a uma salinização do solo e a problemas de absorção de água e nutrientes. De acordo com Guines et al. (1996), uma concentração de sais correspondente a $9.500 \mu \mathrm{S} \mathrm{cm} \mathrm{cm}^{-1}$ foi considerada altamente salina para pimentão.

Sabe-se que existe uma relação entre teor de nutrientes no solo, teor nas fol has e produção. De acordo com Swiader \& Morse (1982), fol has de pimentão com 4,6 $\mathrm{g} \mathrm{kg}^{-1}$ deP apresentavam sintomas visíveis de toxidez, enquanto $2,8 \mathrm{~g} \mathrm{~kg}^{-1}$ de $P$ nas fol has relacionavam-se com as mais altas produções, em condições de campo. J á Lorenz \& Vittum (1980) relataram ser o teor deficiente em $\mathrm{P}$ no pecíolo de folhas expandidas de $2,0 \mathrm{~g} \mathrm{~kg}^{-1}$ eser onível suficiente de $4,0 \mathrm{~g} \mathrm{~kg}^{-1}$. Foi observado que, no florescimento, os teores foliares de $\mathrm{P}$ podem variar entre $1,5 \mathrm{e} 2,5 \mathrm{~g} \mathrm{~kg}^{1}$ do peso da matéria seca.

De acordo com Hassan \& Hamlan (1994), a aplicação de $K$ resultou em maiores teores foliares desse nutriente em pimenta (chilli), sem alterar as concentrações $\left(\mathrm{g} \mathrm{kg}^{-1}\right)$ de N, P, e Ca, que foram, no início da frutificação, respectivamente, de 40,5; 6,9 e 31,0 para $132 \mathrm{~kg} \mathrm{ha}^{-1}$ de $\mathrm{K}_{2} \mathrm{O}$ aplicado. O teor de $\mathrm{K}\left(\mathrm{g} \mathrm{kg}^{-1}\right)$, para a mesma dose, foi de $43,2 \mathrm{~g} \mathrm{~kg}^{-1}$. As concentrações de nutrientes nas fol has e caules determinadas por Hegde (1989), aos 67 dias do transplante, em plena frutificação, foram $\left(\mathrm{g} \mathrm{kg}^{-1}\right)$ : $\mathrm{N}=52,3$ e 20,$2 ; \mathrm{P}=8,1$ e 4,6; $\mathrm{K}=48,8$ e 30,7 ; $\mathrm{Ca}=38,1$ e 16,2; $\mathrm{Mg}=23,8$ e 16,5. 
O pimentão apresentou maior exigência nutricional nos estádios reprodutivos (Miller et al., 1979). Segundo os autores, maior acúmulo de N, P, $\mathrm{K}$, Ca e Mg nas plantas ocorreu no início do aparecimento dos primeiros frutos.

De acordo com Hegde (1988), a absorção dos nutrientes aumentou significativamente com o $\mathrm{N}$ aplicado (doses entre 60 e $180 \mathrm{~kg} \mathrm{ha}^{-1}$, aplicados no início do plantio e após 30 dias), tendo sido as quantidades encontradas na planta intei $\mathrm{ra}\left(\mathrm{kg} \mathrm{ha}^{-1}\right)$, ao final do ciclo de cultivo de N - 55,5; P - 13,2; K 73,1; Ca - 22,3, Mg - 20,9. De acordo com o mesmo autor, o máximo de absorção correspondeu a $180 \mathrm{~kg} \mathrm{ha}^{-1}$ de $\mathrm{N}$ e resultou em um máximo de matéria seca produzida, assim como um máximo número, peso e produção de frutos. Por outrolado, o $\mathrm{N}-\mathrm{NO}_{3}$ - pode ter um efeito antagônico com outros ânions, como o cloreto, provocando a diminuição do elemento absorvido.

Gomes et al . (1996) encontraram diminuição nos teores de cloreto no pimentão, em função da adubação nitrogenada, enquanto a adubação potássica $(\mathrm{KCl})$ teve um efeito inverso, aumentando o cloreto nas plantas.

Os teores de cloreto requeridos pelo pimentão, para um ótimo crescimento, de acordo com Marscher (1997), estão entre 0,2 e 0,4 $\mathrm{mg} \mathrm{g}^{-1}$ na matéria seca, e, em média, as plantas podem conter de 2 a $20 \mathrm{mg} \mathrm{g}^{-1}$ do nutriente na matéria seca; os sintomas detoxidez manifestam-se, em plantas mais sensíveis, quando os teores estão entre 20 e $30 \mathrm{mg} \mathrm{g}^{-1}$. Mills \& J ones (1996) referem-se a níveis de cloreto entre 0,05 e $0,2 \mathrm{mg} \mathrm{g}^{-1}$, comumente encontrados nas plantas.

Considerando a necessi dade de mais informações sobre a nutrição do pimentão em condições controladas, objetivou-se, no presente trabalho, não só avaliar a influência do $\mathrm{N}$ e K na concentração e acúmulo de nutrientes nas fol has e plantas, mas também verficar o efeito da adubação na produção do pimentão e o efeito sal ino do $\mathrm{KCl}$ aplicado ao solo.

\section{MATERIAL E MÉTODOS}

Para avaliar a influência do N e doK na nutrição do pimentão, cultivar Mayata, desenvolveu-se o experimento em ambiente protegido (casa de vegetação com telas laterais e área de $210 \mathrm{~m}^{2}$ ), no Núcleo Experimental, Instituto Agronômico de Campinas (I AC), em L atossoloVermel ho mesoférrico (EMBRAPA, 1999).

A análise química do solo, amostrado em duas profundidades, apresentou, após correção, na camada de $0-20 \mathrm{~cm}: \mathrm{pH}\left(\mathrm{CaCl}_{2}\right)=5,7$; matéria orgânica $=$ $24,0 \mathrm{~g} \mathrm{dm}^{-3} ; \mathrm{P}$ resina $=199 \mathrm{mg} \mathrm{dm}^{-3} ; \mathrm{SO}_{4}=$ $250,7 \mathrm{mg} \mathrm{dm}^{-3} ; \mathrm{N}=1,8 \mathrm{~g} \mathrm{~kg}^{-1} ; \mathrm{K}=3,2 \mathrm{mmol}_{\mathrm{c}} \mathrm{dm}^{-3}$; $\mathrm{Ca}=52,0 \mathrm{mmol}_{\mathrm{c}} \mathrm{dm}^{-3} ; \mathrm{Mg}^{-19}, 0 \mathrm{mmol}_{\mathrm{C}} \mathrm{dm}^{-3} ; \mathrm{H}+\mathrm{Al}$ $=22,0 \mathrm{mmol}_{\mathrm{C}} \mathrm{dm}^{-3}, \mathrm{SB}=74,2 \mathrm{mmol}_{\mathrm{c}} \mathrm{dm}^{-3} ; \mathrm{T}=$
$96,2 \mathrm{mmol}_{\mathrm{c}} \mathrm{dm}^{-3} ; \mathrm{V} \%=77 . \mathrm{Na}$ camada de $20-40 \mathrm{~cm}$, os resultados foram: $\mathrm{pH}\left(\mathrm{CaCl}_{2}\right)=4,8$; matéria orgânica $=24,0 \mathrm{~g} \mathrm{dm}^{-3} ; \mathrm{P}$ resina $=39 \mathrm{mg} \mathrm{dm}^{-3} ; \mathrm{SO}_{4}$ $=134,3 \mathrm{mg} \mathrm{dm}^{-3} ; \mathrm{N}=1,7 \mathrm{~g} \mathrm{~kg}^{-1} ; \mathrm{K}=3,5 \mathrm{mmol}_{\mathrm{c}} \mathrm{dm}^{-3}$; $\mathrm{Ca}=27,0 \mathrm{mmol}_{\mathrm{C}} \mathrm{dm}^{-3} ; \mathrm{Mg}^{-3}=14,0 \mathrm{mmol}_{\mathrm{c}} \mathrm{dm}^{-3} ; \mathrm{H}+\mathrm{Al}$ $=34,0 \mathrm{mmol}_{\mathrm{C}} \mathrm{dm}^{-3}, \mathrm{SB}=45,0 \mathrm{mmol}_{\mathrm{C}} \mathrm{dm}^{-3} ; \mathrm{T}=$ $79,0 \mathrm{mmol}_{\mathrm{c}} \mathrm{dm}^{-3} ; \mathrm{V} \%=57$. O método adotado seguiu a recomendação de Raij et al. (1987).

Aplicaram-se, de acordo com a análise do solo, quantidades relativas a 2,0 $\mathrm{t} \mathrm{ha}^{-1}$ de calcário dolomítico, para corrigir a acidez e elevar o teor de Mg para $9 \mathrm{mmol}_{\mathrm{C}} \mathrm{dm}^{-3}$ e quantidade equivalente a $20 \mathrm{t} \mathrm{ha}^{-1}$ de esterco curtido (Nagai et al., 1995). A caracterização do esterco $\left(\mathrm{g} \mathrm{kg}^{-1}\right)$, com base em umidade natural, foi: $\mathrm{pH}\left(\mathrm{CaCl}_{2}\right)=6,5$; umi dadetotal =410; matéria orgânica total =256; matéria orgânica compostável $=216$; carbono orgânico $=120$; $\mathrm{N}$ total $=5,0 ; \mathrm{P}_{2} \mathrm{O}_{5}$ total $=5,1 ; \mathrm{K}_{2} \mathrm{O}=6,0 ; \mathrm{Ca}$ total $=28,0$; $\mathrm{Mg}$ total $=1,9 ; \mathrm{S}$ total $=1,7$; rel ação $\mathrm{C} / \mathrm{N}=24 / 1$.

A adubação mineral foi feita sete dias antes do transplante das mudas, colocando-se, nos sulcos de plantio, $60 \mathrm{~g} \mathrm{~m}^{-2}$ de $\mathrm{P}_{2} \mathrm{O}_{5}, 100 \mathrm{mg} \mathrm{m}^{-2}$ de $\mathrm{B}$ e $240 \mathrm{mg} \mathrm{m}^{-2}$ de $\mathrm{Zn}$, nas formas de superfosfato simples, bórax e sulfato de zinco. As mudas foram transplantadas em 01/11/96, 50 dias após a semeadura. As adubações, nitrogenada (uréia) e potássica (cloreto de potássio), foram iniciadas 13 dias após o transplante e foram parceladas em seis vezes, entre o período de 13/11/96 a 24/01/97. O período de aplicação dos adubos em cobertura não acompanhou o ciclo da cultura, pois se considerou a alta retenção dos cátions no sol o, decorrente da sua textura e do teor de matéria orgânica.

Os tratamentos compreenderam uma testemunha sem $\mathrm{N}$ e $\mathrm{K}$ e as combinações, em esquema fatorial, de três doses de $\mathrm{N}\left(13,3 ; 26,6\right.$ e $\left.39,9 \mathrm{~g} \mathrm{~m}^{-2}\right)$ e três doses de $\mathrm{K}\left(5,5 ; 11,1\right.$ e $\left.16,6 \mathrm{~g} \mathrm{~m}^{-2}\right)$, totalizando 10 parcelas. As doses intermediárias de $\mathrm{N}$ e $\mathrm{K}$ foram aplicadas de acordo com resultados da análise do solo, seguindo recomendação de Boaretto (1986) e Raij et al. (1996). Os adubos foram aplicados aolado das plantas, nas duas linhas de cada canteiro, logo abaixo dos gotejadores.

A água usada na irrigação foi a mesma que abastecia a fazenda, com uma condutividade elétrica média de $400 \mu \mathrm{S} \mathrm{cm}^{-1}$, e os gotejadores (barras de plástico achatadas e perfuradas), foram colocados ao lado das linhas de plantio, seguindo o comprimento das parcelas. O volume deágua esteve entrea umidade mínima necessária para o pi mentão e a umidade na capacidade de campo, considerando a área da parcela e a profundidade do sistema radicular. $O$ volume de água mínimo limitante para o pimentão, para um potencial matricial $(\psi)$ igual a -11 kPa, foi calculado na curva de retenção de água pelo solo (Wosten \& Genuchten, 1988), sendo de $0,210 \mathrm{~cm}^{3} \mathrm{~cm}^{-3}$ (proporção água/solo). A capacidade máxima de retenção de água foi obtida experimentalmente, de acordo com Pereira (1995). 
O tempo de irrigação das parcelas foi calculado conformea vazão dos gotejadores e o vol ume deágua necessário. A vazão média dos gotejadores foi de $394 \mathrm{~mL}$, por planta, a cada 15 min ou 17,4 L deágua, por parcela por hora. O potencial matricial, correspondenteàs umi dades, foi lido em tensiômetros instalados no solo, nas profundidades de 20 e $40 \mathrm{~cm}$, no início, meio e final da estufa; a leitura nos tensiômetros serviram de base para estabelecer a freqüência de irrigação, variando, conforme o ambiente interno da estufa (temperatura e umidade), de um a três dias. Os cálculos do $(\psi)$, relativo à umidade na capacidade de campo, seguiram Wosten \& Genuchten (1988).

Para a col eta das raízes, adotou-se o método de Bohn (1979), com al gumas modificações introduzidas pelo Centro de Ecofisiologia e Biofísica do IAC, e utilizou-se trado específico, com vol ume de $407 \mathrm{~cm}^{3}$. O peso do solo úmido correspondeu, em média, a $500 \mathrm{~g}$, com umidade entre 25 e $30 \%$, dependendo do dia de col eta. As raízes for am col etadas logo abaixo da linha de gotejo, nas profundidades de 0-10, 10-20 e $20-40 \mathrm{~cm}$. Foram feitas duas amostragens por planta, utilizando três plantas por parcela. O trado foi colocado a uma distância de $10 \mathrm{~cm}$ do caule da planta. Os tratamentos amostrados foram aqueles onde o menor nível de $\mathrm{N}$ estava combinado com o menor e com o maior nível de K, bem como a combinação entreas doses máximas dos nutrientes. O mesmo procedimento foi adotado para a escol ha dos tratamentos com níveis de K, além do controle.

O objetivo destes tratamentos foi verificar a limitação no crescimento das raízes por um possível efeito de salinidade do $\mathrm{KCl}$, para altos níveis de $\mathrm{K}$ adicionado. A separação das raízes do solo foi feita por meio de dispersão em água e fracionamento do solo e pela suspensão e peneiramento das raízes (Fujimura et al., 1994). As raízes foram lavadas, secas sobre papel-toal ha efez-sea separação das impurezas, como raízes de ervas daninhas e material orgânico.

A condutividade elétrica foi determinada após a col heita dos frutos, utilizando-se uma proporção soloágua de $1: 1$ (50 g de solo e $50 \mathrm{~mL}$ de água deionizada). Após homogeneização e $24 \mathrm{~h}$ em repouso, a suspensão foi centrifugada por 20 min e lida em condutivímetro (Camargo et al.,1986).

Para determinar a condutividade elétrica (CE), foram amostrados os tratamentos relativos à duas doses de $\mathrm{N}\left(13,3\right.$ e 39,9 $\left.\mathrm{g} \mathrm{m}^{-2}\right)$ e duas doses de $\mathrm{K}(5,5$ e $16,6 \mathrm{~g} \mathrm{~m}^{-2}$ ), além do controle, sem os nutrientes, totalizando cinco parcelas.

$O$ delineamento experimental adotado foi o de blocos ao acaso, em esquema fatorial $(3 \times 3+1)$, com quatro repetições, totalizando 40 parcelas. Cada parcela experimental foi composta de 11 plantas, dispostas em duas linhas, num total de 444 plantas. Foram consideradas úteis as sete plantas em posi ção central, ocupando, cada planta, uma área de 0,30 m², para 0,50 m entre plantas e 0,60 m entrelinhas.
Noinício do florescimento, 50 dias do transplante, e no início da frutificação, 83 dias do transplante, retiraram-se fol has recém-maduras, considerando a terceira ou quarta folha a partir do ápice, para a determinação do teor de nutrientes. Foram retiradas de quatro a cinco fol has por planta de cinco a seis plantas por parcela.

Para avaliar a produção, foram col hidos os frutos recém-maduros ou no ponto de consumo, durante 34 semanas, totalizando 18 colheitas. Em cada colheita, os frutos foram medidos e pesados. Ao final do experimento, foram colhidas sete plantas por parcela, separando-se caule, folhas e frutos remanescentes. As amostras defol has, caules efrutos, recém-maduros (parteaérea), após secagem em estufa a $65^{\circ} \mathrm{C}$, até peso constante, foram moí das eanalisadas para a determinação dos teores de macronutrientes de acordo com o método descrito por Bataglia et al. (1983), tendo a determinação do S seguido método proposto por Vitti (1989). Os frutos para a determinação dos teores de nutrientes, foram col hidos ao longo do ciclo, em quatro col heitas (cinco frutos por parcela, por col heita). O acúmulo dos nutrientes nos frutos foi calculado ao final do ciclo, de acordo com os teores determinados nas col heitas anteriores.

Para avaliar a produção de matéria seca da parte aérea e matéria seca da raiz, a concentração e o acúmulo de nutriente, assim como a produção de frutos, utilizou-se fatorial $3 \times 3$ (Gomes, 1987). Para avaliar a condutividade elétrica, utilizou-se fatorial $2 \times 2$. Para as mesmas variáveis, utilizou-se o teste de Dunnett bilateral a 5\% para comparar a testemunha com os demais tratamentos.

\section{RESULTADOS E DISCUSSÃO}

\section{Teores de nutrientes nas folhas}

Os teores médios foliares de nutrientes $\left(\mathrm{g} \mathrm{kg}^{-1}\right)$, determinados em fol has recém-maduras de pimentão, no início do florescimento, foram, em média: $\mathrm{N}=54,3 ; \mathrm{P}=2,3 ; \mathrm{K}=64,9 ; \mathrm{Ca}=10,4 ; \mathrm{Mg}=$ 5,3; $S=4,2$ e, no início da frutificação, foram, em média, de $\mathrm{N}=48,7 ; \mathrm{P}=2,7 ; \mathrm{K}=66,8 ; \mathrm{Ca}=15,4 ; \mathrm{Mg}$ $=7,0 ; S=4,4$. Os tratamentos não influenciaram, de forma significativa, os teores de nutrientes nas fol has do pimentão.

Comparados com os teores foliares, citados por Reuter \& Robison (1997), concluiu-se que a cultura esteve adequadamente suprida de nutrientes, exceto para o $\mathrm{P}$, que se encontrava próximo ao nível crítico. Esses autores consideraram adequados ou suficientes, para plantas no início do florescimento, teores médios ( $\mathrm{g} \mathrm{kg}^{-1}$ ) entre: $\mathrm{N}=40,0-60,0 ; \mathrm{P}=3,5$ 10,$0 ; \mathrm{K}=40,0-60,0 ; \mathrm{Ca}=10,0-25,0 ; \mathrm{Mg}=3,0-10,0$. Teores semelhantes foram reportados por Mills \& $J$ ones (1996), em plantas produzindo os primeiros frutos: $\mathrm{N}=35,0$ a 50,$0 ; \mathrm{P}=2,2$ a 7,$0 ; \mathrm{K}=35,0$ a 45,0; $\mathrm{Ca}=13,0$ a 28,$0 ; \mathrm{Mg}=2,5$ a 12,0 . 
A aplicação de altas doses de $\mathrm{K}\left(16,6 \mathrm{gm}^{-2}\right)$ diminuiu significativamentea concentração de $\mathrm{P}$ nas folhas, quando a amostragem foi feita no início da frutificação (83 dias após o transplante). Doses de $\mathrm{K}$, como $\mathrm{KCl}$, de 16,6 $\mathrm{g} \mathrm{m}^{-2}$, dificultaram a absorção de $\mathrm{P}$, decorrente da menor produção de raízes (Quadro 1), provavelmenteem razão do efeito salino do $\mathrm{KCl}$. A pós o aparecimento dos primeiros frutos, doses de $16,6 \mathrm{~g} \mathrm{~m}^{-2}$ deK diminuíram significativamente o teor de S nas fol has, de 4,60 para 4,05 $\mathrm{g} \mathrm{kg}^{-1}$, principalmente na dose intermediária de $\mathrm{N}$, possivelmente pelo antagonismo entre sulfato e cloreto.

O N, K, Ca e Mg não tiveram diminuição na concentração foliar, até o início da frutificação, evidenciando maior crescimento da planta a partir desse estádio. Resultados semel hantes foram encontrados por K navel (1977), em cultivo protegido, assim como Locascio \& Alligood (1992), Hassan et al. (1993); Locascio \& Stall (1994), em cultivo de campo, verificaram que a concentração foliar de $\mathrm{N}$ somente diminuiu a partir do aparecimento dos primeiros frutos, o que foi atribuído ao maior crescimento das plantas de pimentão a partir dessa época. Locascio \& Fiskell (1979) relataram menores concentrações foliares de NPK com a adubação nitrogenada.

Hochmuth et al. (1988), em Martin e Palm Beach, Flórida, cultivando pimentão em condições de campo em solos mais arenosos, encontraram, em folhas recém-maduras, no início do florescimento, teores de $\mathrm{K}$ entre 57 e $72 \mathrm{~g} \mathrm{~kg}^{-1}$. Porém, somente depois de um mês das primeiras amostragens (início da frutificação) é que foram verificadas diminuições

Quadro 1. Produção de matéria seca de raízes de pimentão submetido a doses de $\mathbf{N}$ (uréia) e K ( $\mathrm{KCl})$ em cobertura, em relação à testemunha, após 34 semanas de cultivo em ambiente protegido

\begin{tabular}{|c|c|c|c|c|}
\hline \multirow{2}{*}{$\mathbf{N}$} & \multirow{2}{*}{$\mathbf{K}$} & \multicolumn{3}{|c|}{ Profundidade $(\mathrm{cm})$} \\
\hline & & 0-10 & $10-20$ & $20-40$ \\
\hline \multicolumn{2}{|c|}{$-\mathrm{g} \mathrm{m}^{-2}$} & \multicolumn{3}{|c|}{ g por amostragem(3) } \\
\hline 0,0 & 0,0 & 0,37 & 0,22 & 0,04 \\
\hline 13,3 & 5,5 & $0,59 * *(1)$ & $0,42 * *$ & $0,12 * *$ \\
\hline 13,3 & 16,6 & $0,45^{n s(2)}$ & $0,26^{\text {ns }}$ & $0,05^{\text {ns }}$ \\
\hline 39,9 & 5,5 & $0,54 *(1)$ & $0,40 * *$ & $0,11^{*}$ \\
\hline 39,9 & 16,6 & $0,49^{\text {ns }}$ & $0,27^{\mathrm{ns}}$ & $0,09 *$ \\
\hline \multicolumn{2}{|c|}{ Média } & 0,49 & 0,31 & 0,08 \\
\hline \multicolumn{2}{|c|}{ C.V. (\%) } & 17 & 17 & 31 \\
\hline
\end{tabular}

(1) Diferenças significativas a 5\% (*) e 1\% (**). (2) Não-significativas, entre as médias dos tratamentos em relação à testemunha, pelo teste de Dunnett. ${ }^{(3)}$ Peso da matéria seca da raiz de seis plantas por parcela. significativas nas concentrações foliares de $K$, quando onutrientefoi aplicadono solo, provavel mente por causa do mai or crescimento das plantas a partir dessa época.

\section{Acúmulo de nutrientes nos órgãos da planta}

As adubações, nitrogenada e potássica, tiveram pouca influência nos teores de nutrientes nas fol has, porém influenciaram, de forma significativa, o acúmulo de nutrientes, ao final do ciclo de cultivo, após 34 semanas. Foi observado que a dose intermediária de $\mathrm{N}\left(26,6 \mathrm{gm}^{-2}\right)$, correspondente a $270 \mathrm{~kg} \mathrm{ha}^{-1}$, comparativamente à testemunha, propiciou o mai or acúmulo de nutrientes pela parte aérea do pimentão (caule + fol has e frutos) (Figura 1). Observou-se maior acúmulo de $\mathrm{N}, \mathrm{K}$ e Ca, obedecendo a uma relação quadrática. Os resultados podem ser explicados pelo maior crescimento da planta e maior produção de matéria seca (Quadro 2). Doses de $\mathrm{N}$ maiores que $26,6 \mathrm{~g} \mathrm{~m}^{-2}$ diminuíram os nutrientes acumulados, o que pode ter ocorrido pela inibição competitiva entre formas do $\mathrm{N}$, decorrentes da transformação da uréia, com os cátions e ânions do solo.

$$
\begin{array}{|c|c|}
\hline & \text { P y }=3,90+0,196 x-0,004 x^{2} \\
\circ & \text { Ca } y=3,97+0,435 x-0,007 x^{2} \\
\Delta & \text { Mg } y=1,23+0,296 x-0,005 x^{2} \\
\circ & \text { S y }=4,21+0,201 x-0,004 x^{2} \\
\times & \text { N y }=41,66+1,036 x-0,022 x^{2} \\
& \text { K y }=55,91+1,827 x-0,035 x^{2}
\end{array}
$$

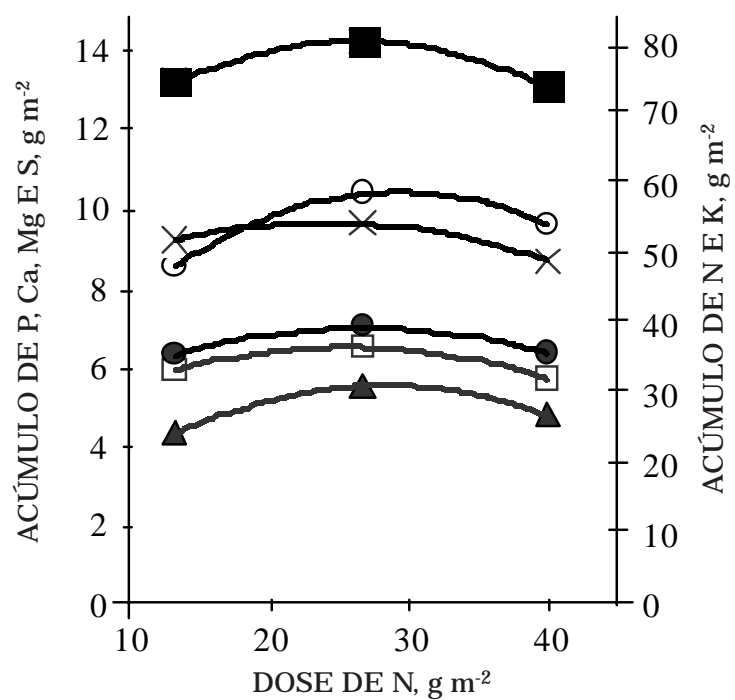

Figura 1. Acúmulo de nutrientes pela parte aérea do pimentão (caule, folhas e frutos), considerando a aplicação de $\mathbf{N}$ no solo, na dose de $\mathrm{K}$ de $5,5 \mathrm{~g} \mathrm{~m}^{-2}$, após 34 semanas de cultivo em ambiente protegido. 
Thomas \& Heilman (1967), trabalhando com o cultivar Yol o Wonder, Hegde (1988) e Locascio et al . (1985), com o cultivar California Wonder, e Crespo Ruiz et al. (1988), com o cultivar Cubanelle, observaram que doses de $\mathrm{N}$ maiores que $120 \mathrm{~kg} \mathrm{ha}^{-1}$ não influenciaram a quantidade de nutrientes acumulados pelo pimentão, ao final do ciclo de cultivo, que variou de nove a 14 semanas. Por outro lado, Olsen et al. (1993) encontraram, após 14 semanas do transplante, um máximo de nutrientes absorvidos, relacionados com 280 e $200 \mathrm{~kg} \mathrm{ha}^{-1}$ de N eK aplicados ao solo.

O N favorece o acúmulo de nutrientes, porque aumenta a produção de biomassa do pimentão, uma vez que estimula o crescimento vegetativo, como já demonstrado por muitos autores (Grainfenberg et al., 1985; Hegde, 1987; Manchanda \& Singh, 1988; Hassan et al., 1993; Mishriky \& Alphonse, 1994).

Há algumas variações nos resultados sobre adubação e exigências nutricionais do pimentão encontrados na literatura. Assim, Rinchon et al. (1995), trabalhando com o cultivar Lamuyo, em ambiente protegido, observaram que a parte aérea acumulou $\left(\mathrm{g} \mathrm{m}^{-2}\right)$, respectivamente, 30; 3; 38; 12 e 6 de N, P, K, Ca e Mg, enquanto Graifenberg et al. (1985), também em ambiente protegido, encontraram um acúmulo dos mesmos nutrientes (g/planta), em dois cultivares, YoloWonder e Heldor F1, respectivamente, de 6,7 e 10,0; 0,9 e 1,3; 8,3 e 12,$5 ; 6,2$ e 9,0 e 0,2 e 1,2. São muitos os fatores responsáveis pelas variações nos resultados, como, por exemplo a produtividade, associada ao número de semanas de cultivo, bem como o cultivar empregado e as condições de ambiente de cultivo.
A produção defrutos de pimentão, por outrolado, não esteve relacionada com o maior acúmulo de nutrientes. Em média, o número e a produção de frutos foram de 34,4 e $6,07 \mathrm{~kg} \mathrm{~m}^{-2}$, independentemente dos níveis de $\mathrm{N}$ ou $\mathrm{K}$ aplicados. $\mathrm{E}$ possível que o al to teor de matéria orgânica $\left(24,0 \mathrm{~g} \mathrm{dm}^{-3}\right)$ e o teor médio de $\mathrm{K}\left(3,2 \mathrm{mmol}_{\mathrm{C}} \mathrm{dm}^{-3}\right)$, iniciais do solo, tenham contribuído para os resultados.

O K contribuiu para a absorção de nutrientes somente quando aplicado na menor dose $\left(5,5 \mathrm{~g} \mathrm{~m}^{-2}\right)$, provavelmente pelo efeito salino do $\mathrm{KCl}$, que diminuiu o crescimento das raízes (Quadro 1) e da parte aérea (Quadro 3), diminuindo, conseqüentemente, a absorção e o acúmulo de nutrientes (Figura 2).

O pimentão é uma cultura moderadamente sensível à salinidade (Cruciani, 1980). O efeito salino é determinado por meio da condutividade elétrica (CE ) da solução do solo. Nesse caso, observou-se que a aplicação de $\mathrm{KCl}$ na dose de $16,6 \mathrm{~g} \mathrm{~m}^{-2}$ relacionouse com altos val ores de CE (Figura 3), como 1.000 e $1.300 \mu \mathrm{S} \mathrm{cm}^{-1}$ a 20 e $40 \mathrm{~cm}$ de profundidade.

Plantas de pimentão cresci das em concentrações de $\mathrm{KCl}$ crescentes entre 4.000 e $10.000 \mu \mathrm{S} \mathrm{cm}^{-1}$, tiveram diminuição no crescimento e diminuição do tamanho e peso dos frutos, provavel mente decorrente do aumento da força iônica na solução nutritiva. O caule acumulou mais assimilados que os frutos, havendo menor consumo deágua; as fol has apresentaram diminuição do potencial hídrico e os frutos tiveram comprometimento da sua firmeza (Tadesse et al., 1999). Os mesmos autores relacionaram o aumento da salinidade do $\mathrm{KCl}$, em solução nutritiva, após 10 semanas do florescimento,

Quadro 2. Produção de matéria seca das plantas de pimentão submetidas a doses de N (uréia), após 34 semanas de cultivo em ambiente protegido

\begin{tabular}{lcc}
\hline Matéria seca na planta & E quação & $\mathbf{R}^{\mathbf{2}}$ \\
\hline Caule & $\mathrm{Y}=0,230892+0,0012012 \times$ & 0,82 \\
Folha & $\mathrm{Y}=0,110478+0,0008058 \mathrm{x}$ & 0,72 \\
Caule +Folha & $\mathrm{Y}=0,341356+0,0020076 \mathrm{x}$ & 0,78 \\
\hline
\end{tabular}

Quadro 3. Produção de matéria seca das plantas de pimentão, submetidas a doses de $\mathrm{K}(\mathrm{KCl})$, após 34 semanas de cultivo em ambiente protegido

\begin{tabular}{lll}
\hline Matéria seca na planta & Equação & $\mathbf{R}^{\mathbf{2}}$ \\
\hline Caule & $Y=0,509142-0,0174265 \times+0,00026328 x^{2}$ & 1,00 \\
Fol ha & $Y=0,193694-0,0023179 \times 0,91$ & 0,91 \\
Caule + Folha & $Y=0,760367-0,0249271 \times+0,00036056 x^{2}$ & 1,00 \\
\hline
\end{tabular}




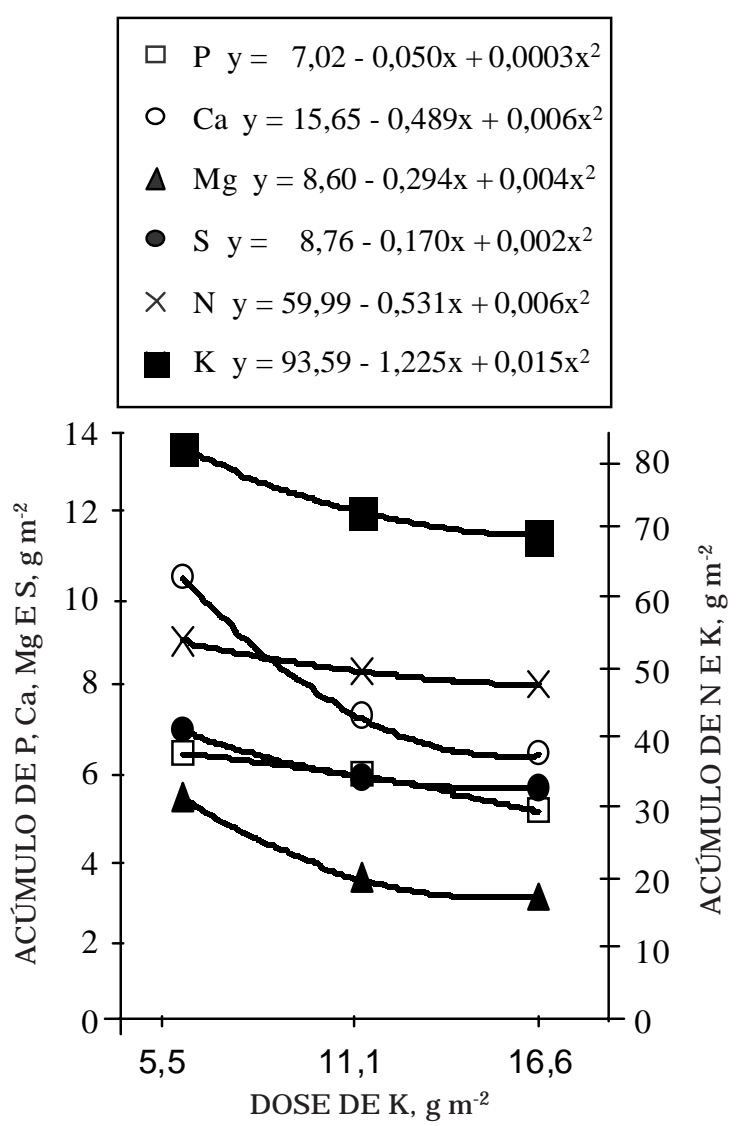

Figura 2. Acúmulo de nutrientes pela parte aérea do pimentão (caule, folhas e frutos), considerando a aplicação de $\mathrm{K}$ no solo, na dose de $\mathbf{N}$ de $26,6 \mathrm{~g} \mathrm{~m}^{-2}$, após 34 semanas de cultivo em ambiente protegido.

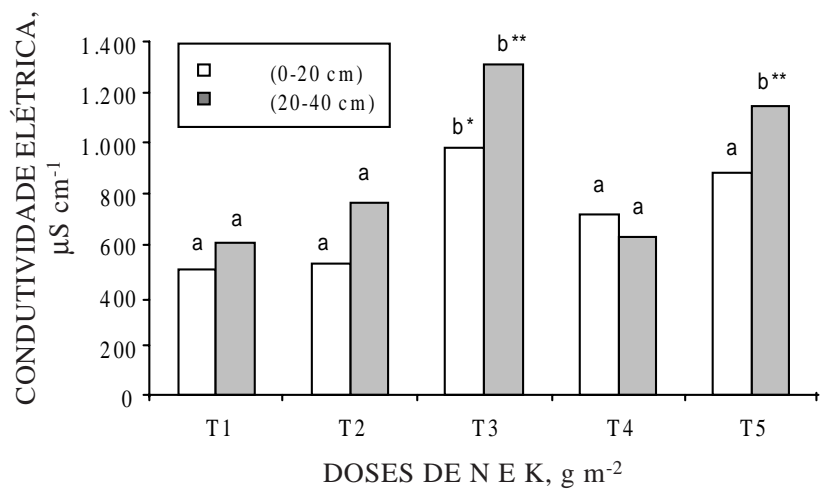

Figura 3. Condutividade elétrica do solo cultivado com pimentão, após 34 semanas, considerando a profundidade de amostragem e as doses de $\mathbf{N}$ e K aplicados ao solo. Para a mesma profundidade, barras seguidas da mesma letra não diferem significativamente pelo teste de Dunnett a 5 (*) e 1 (**)\%. T1- testemunha sem $\mathbf{N}$ e K; T2- N = 13,3 e K =5,5 $\mathrm{g} \mathrm{m}^{-2}$; T3 - N =13,3 e K = 16,6 $\mathrm{g} \mathrm{m}^{-2}$; T4 - N = 39,9 e K = 5,5 $\mathrm{g} \mathrm{m}^{-2}$; T5 - N = 39,9 e K = $16,6 \mathrm{~g} \mathrm{~m}^{-2}$.
Quadro 4. Teores de cloro em plantas de pimentão, submetido a doses de $\mathbf{N}$ (uréia) e $\mathrm{K}(\mathrm{KCl})$ em cobertura, após 34 semanas de cultivo em ambiente protegido

\begin{tabular}{cccc}
\hline N e K & Caule & Folha & Fruto \\
\hline g m$^{-2}$ & \multicolumn{3}{c}{ mg kg-1 $^{-1}$} \\
$\mathrm{~N} 13,3$ & 5.140 & 8.050 & 4.302 \\
26,6 & 4.810 & 7.160 & 4.036 \\
39,9 & 4.480 & 7.270 & 3.860 \\
$\mathrm{~K} 5,5$ & 4.630 & 7.360 & 3.775 \\
11,0 & 5.060 & 7.680 & 4.022 \\
16,6 & 4.740 & 7.430 & 4.400 \\
$\mathrm{~F}(\mathrm{~N})$ linear & $*$ & $\mathrm{~ns}$ & $*$ \\
$\mathrm{~F}(\mathrm{~K})$ & $\mathrm{ns}$ & $\mathrm{ns}$ & $*$ \\
C.V. (\%) & 13,9 & 24,5 & 9,5 \\
\hline
\end{tabular}

(1) Diferenças significativas a $5 \%(*)$, entre as médias dos tratamentos, pelo teste de Tukey.

com a diminuição na concentração de Ca e Mg e aumento na concentração de $\mathrm{K}$ nos frutos, considerando a redução do tamanho e peso do pimentão. Richter et al. (1999) encontraram efeito salino para pimentão, em valores de condutividade elétrica menores que $1.460 \mu \mathrm{S} \mathrm{cm}^{-1}$, causando redução no tamanho e rendimento dos frutos.

$O$ efeito do $\mathrm{KCl}$ noaumento da $\mathrm{CE}$ ena salinidade do solo com pimentão foi relatado por Bernstein \& Francois (1975), após nove semanas de cultivo.

Observou-se que os teores de $\mathrm{Cl}$ na parte aérea do pimentão (Quadro 4) estão acima dos teores requeridos para o ótimo crescimento da cultura, de acordo com Marschner (1997). Os teores no caule e fol has estão abaixo dos teores referidos por Sziklai et al. (1988), enquanto os frutos tiveram concentrações semel hantes do nutriente encontrado no presente trabal ho. Todavia, as altas quantidades de $\mathrm{Cl}$ absorvidas pelo pimentão não causaram sintomas de toxidez visíveis nas folhas, embora, de acordo com Silva et al. (1999), a produção tenha diminuído de 39 para 32 frutos $\mathrm{m}^{-2}$ e o rendimento diminuído de 6,8 para $5,7 \mathrm{~kg} \mathrm{~m}^{-2}$, quando o $\mathrm{K}$, como $\mathrm{KCl}$, foi aplicado na dose de $16,6 \mathrm{~g} \mathrm{~m}^{-2}$.

Os nutrientes acumulados nos frutos não tiveram influência da adubação nitrogenada, o que concorda com os resultados de Hochmuth et al. (1994), embora Locascio et al. (1985), Crespo Ruiz et al. (1988) e Olsen et al. (1993) tenham demonstrado o contrário. O pimentão acumulou nos frutos, em média $\left(\mathrm{g} \mathrm{m}^{-2}\right)$, 45,0; 5, 7; 58,0; 1,9; 1,5;4,8, respectivamente, de N, P, $\mathrm{K}, \mathrm{Ca}, \mathrm{MgeS}$.

Em relação ao total, o caule acumulou maior quantidade de $\mathrm{Mg}$, $\mathrm{Ca}$, S e K (33; 30; 16 e 11\%, respectivamente); as fol has contribuíram mais com o Ca e Mg (51 e 39\%, respectivamente); os frutos contiveram mais P, N, K e S (90; 84; 78 e 73\%, respectivamente) (Figura 4). 


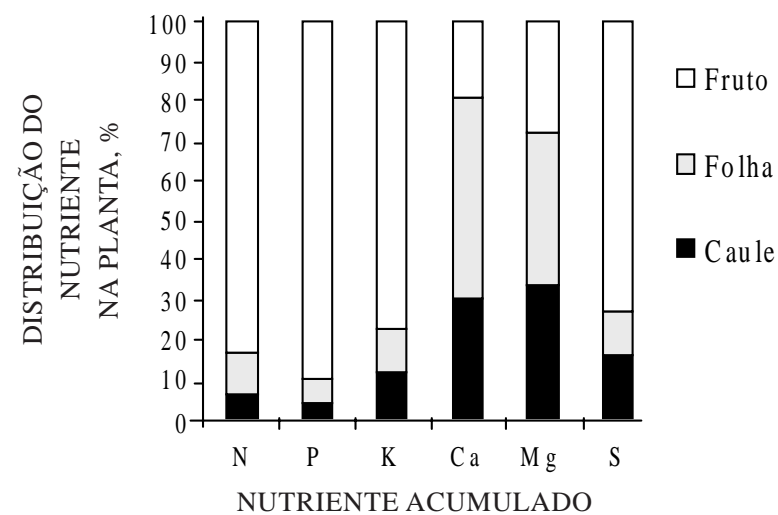

Figura 4. Distribuição percentual dos nutrientes acumulados na parte aérea do pi mentão (caule, folhas e frutos), após 34 semanas de cultivo em ambiente protegido eaplicação de $\mathbf{N}$ e K ao solo.

A distribuição dos nutrientes encontrada por Miller et al. (1979) foi de: N (21, 36 e 44\%); K (25, 35 e 40\%); P (38, 21 e 41\%); Ca (29, 64 e $6 \%) ; M g(34,50$ e $16 \%)$, respectivamente, para caules, fol has efrutos. As fol has apresentaram mai or percentagem de Ca e $\mathrm{Mg}$, enquanto os frutos continham mais $\mathrm{N}, \mathrm{P}$ eK. O $K$ distribuiu-se em partes iguais nos frutos e demais órgãos vegetativos. A distribuição dos nutrientes nos frutos concorda com Olsen et al. (1993) e nas folhas e frutos concorda com Miller et al. (1979).

Considerando as variações que ocorreram em trabalhos citados por outros autores, pode-se dizer que, de forma geral, os resultados de distribuição percentual de nutrientes nos diferentes órgãos da planta de pimentão encontrados neste trabalho concordam com os dados de literatura.

\section{CONCLUSÕES}

1. As doses de $\mathrm{N}$ não afetaram a concentração dos nutrientes presentes em fol has recém-maduras, enquanto o $\mathrm{K}$ diminuiu a concentração de $\mathrm{P}$ e $\mathrm{S}$, no início da frutificação.

2. O N aumentou a quantidade de nutrientes no caule, fol has e parte aérea, ao final do ciclo, quando aplicado na dose de $26,6 \mathrm{~g} \mathrm{~m}^{-2}$. O K ajudou na absorção de nutrientes, somente quando presente em baixas doses $\left(5,5 \mathrm{~g} \mathrm{~m}^{-2}\right)$ no solo.

3. Os nutrientes acumulados pelos frutos não foram influenciados pel os tratamentos e a produção dos frutos de pimentão não foram influenciados pela aplicação do N eK no solo.

4. $\mathrm{O} \mathrm{K}$ como $\mathrm{KCl}$, na dose de $16,6 \mathrm{~g} \mathrm{~m}^{-2}$, causou problemas de salinidade no solo.

\section{AGRADECIME NTOS}

Ao Engenheiro-Agrônomo Flávio Pagani de Castro e ao Sr. Assis Lopes dos Santos, pelo apoio nos trabalhos de campo, e ao Prof. Dr. Takashi Muraoka, pela amizade e ensinamentos, que muito contribuíram para a realização e êxito deste trabalho.

\section{LITERATURA CITADA}

BATAGLIA, O.C.; FURLANI, A.M.C.; TEIXEIRA, J.P.F.; FURLANI,P.R.\& GALLO, J.R. Métodos de análise química de plantas. Campinas, Instituto Agronômico, Campinas, 1983. 48p. (Bol etim Técnico, 78)

BERNSTEIN, L.; FRANCOIS, E. Effects of frequency of sprinkling with saline water comparated whith daily dripirrigation. Agron. J ., 67:185-190, 1975.

BOARETTO, A.E. Análise química de terra e recomendação de calagem e adubação para as principais culturas do estado de São Paulo. Botucatu, Fundação de Estudos Agrícolas e Florestais, 1986. 50p. (Boletim Didático, 2)

BOHN, W. Methods of studying root systems. Berlin, SpringerVelag, 1979. 189p.

CAMARGO, O.A.; MONIZ, A.C.;J ORGE, J .A. \& VALADARES, J . M.A.S. Métodos de análise química, mineralógica e física de sol os do I nstituto Agronômico de Campinas. Campinas, Instituto Agronômico de Campinas, 1986, 93p. (Boletim Técnico, 106)

CRESPO RUIZ, M.; GOYAL, M.; BAEZ, C.C. \& RIVIERA, L.E. Nutrient uptake and growth characteristics of nitrogen fertigated sweet peppers under drip irrigation and plastic mulch. J. Agr. Univ. Puerto Rico, 72:575-585, 1988.

CRUCIANI, D.E. A drenagem na agricultura. São Paulo, N obel, 1980. 333p.

EMPRESA BRASILEIRA DE PESQUISA AGROPECUÁRIA EMBRAPA. Centro Nacional de Pesquisa do Solo. Sistema Brasileiro de Classificação do Solo. Rio de J aneiro, 1999. $412 \mathrm{p}$.

FUJ IMURA, M.; KURACHI, S.A.H.; ARRUDA, F.B. \& PIRES, R.C.M. A técnica de estudo de raízes pelo método do trado. Campinas, Instituto Agronômico de Campinas, 1994. 10p. (Boletim Técnico, 153)

GOLLIFER, D.E. Effects of applications of mulch and potassium on Capsicum annuum. Papua New Guinea J . Agric. Forest. Fish., 36:22-29, 1993.

GOMES, F.P. A estatística moderna na pesquisa agropecuária. 2.ed. Piracicaba, Potafos, 1987. 162p.

GOMES, I.; PEDRENÕ, J .N.; MORAL, R.; IBORRA, M.R.; PALACIOS, G. \& MATRIX, J. Salinity and nitrogen fertilization affecting the macronutrient content and yield of sweet pepper plants. J. Plant Nutrit., 19:353-359, 1996. 
GRAIFENBERG, A.; PETSAS, S. \& LENZI, I. Crescita e asportazione degli elementi nutritivi nel peperone allevato in serra fredda. Colt. Prot., 12:33-38, 1985.

GUINES, A.; INAL, A. \& ALPASLAN, M. Effect of salinity on stomatal resistance, proline and mineral composition of pepper. J. Plant Nutr., 19:389-396, 1996.

HASSAN S.A. \& RAMLAN, Z. A. I nfluence of potassium fertilizer and mulching on growth and yield of chilli (Capsicum annuum L.). Acta Hortic., 369:311-318, 1994.

HASSAN, S.A.; GERBER, J.M. \& SPLITTSTOESSER, W.E. Growth and yield potential of green pepper as affected by nitrogen at transplanting. Pert. J. Trop. Agric. Sci., 16:101105, 1993.

HEGDE, D.M. I rrigation and nitrogen requirement of bell pepper (Capsicum annuum). Ind. J . Agr. Sci., 58:668-672, 1988.

HEGDE, D.M. Effect of soil moisture and nitrogen on plant water relations, mineral composition and productivity of bell pepper (Capsicum annuum L.). Ind. J . Agric., 34:30-34, 1989.

HEGDE, D.M. Growth analysis of bell pepper (Capsicum annuum L.) in relation to soil moisture and nitrogen fertilization. Sci. Hortic., 33:179-187, 1987.

HOCHMUTH, G.J .; SHULER, K.; HANLON, E \& \& ROE, N. Pepper response to fertilization with soluble and controlled rel ease potassium fertilizers. Proc. Fla. St. Hortic., Soc. 107:132-139, 1994.

HOCHMUTH, J.G.; SHULER, K.D.; GILREATH, P.R. \& MITCHELL, R.L. Field testing of revised Mehlich-1 predicted potassium fertilizer recommendation for mulched pepper. Soil Crop Sci. Soc. Fla. Ann. Proc., 47:30-35, 1988.

KNAVEL, D.E. The influences of nitrogen on pepper transplant growth and yielding of plants grown with different levels of soil nitrogen. J. Am. Soc. Hortic. Sci., 102:533-535, 1977.

LOCASCIO, S.J . \& FISKELL, J .G.A. Pepper response to sulphurcoated urea, mulch and nitrogen rate. Proc. Fla. State. Hortic. Soc., 92:112-115, 1979.

LOCASCIO, S.J . \& ALLIGOOD, M.R. Nitrogen and potassium source and N-rate for drip-irrigated pepper. Proc. Fla. State. Hortic. Soc., 105:323-325, 1992.

LOCASCIO, S.J .; FISKELL, J.G.A.; GRAETZ, P.A. \& HAUCK, R.D. Nitrogen accumulation by pepper as influenced by mulch and time of fertilizer application. J. Am. Soc. Hortic. Sci., 110:315-318, 1985.

LOCASCIO, S.J . \& STALL, W.M. Bell pepper yield as influenced by plant spacing and row arrangement. J. Am. Soc. Hortic. Sci., 119:899-902, 1994.

LORENZ, O.A. \& VITTUM, M.T. Phosphorus nutrition of vegetables crops and sugar beets. In: KHASAWNEH, F.E.; SAMPLE, E.C. \& KAMPRATH, E.J., eds. The role of phosphorus in agriculture. Madison, American Society of Agronomy, 1980. p.737-762.

MANCHANDA, A.K. \& SINGH, B. Effect of plant density and nitrogen on growth and fruit yield of bell pepper (Capsicum annuum L.). Ind. J . Agr., 33:445-447, 1988.
MARSCHNER, H. Mineral nutrition of higher plants. 2.ed. San Diego, Academic Press, 1997. 889p.

MILLER, C.H.; McCOLLUM, R.E.\& CLAIMON, S. Relationships between growth of bell peppers (Capsicum annuum L.) and nutrient accumulation during ontogeny in field environments. J. Am. Soc. Hortic. Sci., 104:852-857, 1979.

MILLS, H.A. \& J ONES J r., B. Plant analysis handbook: II. A practical sampling, preparation, analysis and interpretation guide. Athens, MicroMacro Publishing, 1996. 422p.

MISHRIKY, J .F. \& ALPHONSE, M. Effect of nitrogen and plant spacing on growth, yield and fruit mineral composition of pepper (Capsicum annuum L.). B. Fac. Agric. Univ. Cairo, 45:413-431, 1994.

MORALES-PAYAN, J.P.; SANTOS B.M.; STALL. W.M. \& BECWICK, T.A. Interference of purple nutsedge (Cyperus rotundus) population densities on bell pepper (Capsicum annuum) yield influenced by nitrogen. Weed Techinid., 12:230-234, 1998.

MOURA, W.D.; CASALI, V.W.D.; CRUZ, C.D. \& LI MA, P.C. Genetic divergence of phosphorous nutrition efficiency in sweet pepper lines. Pesq. Agropec. Bras., 34:217-224, 1999.

NAGAI, H. Pimentão. In: FAHL, J .I.; CAMARGO, M.B.P.; PIZZINATTO, M.A.; FURLANI, A.M.C.; BETTI, J.A.; MELLO, A.M.T. \& MARIA, I.C., eds. Instruções agrícolas para o estado de São Paulo. 6.ed. Campinas, Instituto Agronômico de Campinas, 1995. p.335-337. (Boletim, 200)

OLSEN, J.K.; LYONS, P.J . \& KELLY, M. Nitrogen uptake and utilization by bell pepper in subtropical Australia. J. Plant. Nutr., 16:177-193, 1993.

PEREIRA, E.C. Avaliação do crescimento e produtividade de pimentão cultivado em diferentes potenciais matriciais de água no solo, em condições de casa de vegetação. Botucatu, Universidade Estadual Paulista, 1995. 64p. (Tese de Mestrado)

RAIJ , B. van; CANTARELLA, H.; QUAGGIO, J .A.; FERREIRA, M.E.; LOPES, A.S. \& BATAGLIA, O.C. Análise química do solo para fins de fertilidade. Campinas, Fundação Cargill, 1987. 170p.

RAIJ , B. van; CANTARELLA, H.; QUAGGIO, J .A. \& FURLANI, A.M.C. Recomendação de adubação e cal agem para o estado deSão Paulo. Campinas, InstitutoAgronômico de Campinas, 1996. 285p.

REUTER, D.J. \& ROBINSON, J.B. Plant analysis: a interpretation manual. 2.ed. Callingwood, CSIRO, 1997. $572 p$.

RICHTER, C.; HEILIGTAG, B.; NAGIEB, M. \& MUKHALLALATI, $U$. Salinity effect in grown and nutrient content of eggfruit (Melongena solanum L.) and sweet pepper (Capsicum annuum L.). Tropenlandwirt, 100:3-16, 1999.

RINCHON, L.; SAEZ, J.; BALSALOBRE, E. \& PELLICER, C. Crecimiento y absorcion de nutrientes del pimiento grueso en cultivo bajo invernadero. Invest. Agr. Prod Prot. Veg., 10:47-49, 1995 
SILVA, M.A.G.; BOARETTO,A.E.; MELO,A.M.T.; FERNANDES, H.G. \& SCIVITTARO, W.B. Rendimento e qualidade de frutos de pimentão cultivado em ambiente protegido em função do nitrogênio e potássio aplicados em cobertura. R. Sci. Agric., 56:1199-1207, 1999. (Suplemento)

SIVIERO, P. \& GALLERANI, M. La cultivazione del pepperone. Verona, Edizioni. L'informatore Agrario, 1992. 217p.

SZIKLAI, I.L.; ÖRDÖGH, M.; MOLNÁR, E. \& SZABÓ, E. Distribution of trace and minor elements in Hungarian spice paprika plants. J . Radioanal. Nuclear Chem., 122:233238, 1988.

SWIADER, J.M. \& MORSE, R.D. Phosphorus solution concentration for production of tomate, pepper and eggplant in minesoils. J. Am. Soc. Hortic. Sci., 107:1149-1153, 1982.

TADESSE, T.; NICHOLS, M.A. \& FISHER, K.J. Nutrient condutivity effects in sweet pepper using a nutrient film technique 1. Yield and fruit quality. New Zealand J . Crop Hortic. Sci., 27:229-237, 1999.
TADESSE, T.; NICHOLS, M.A. \& FISHER, K.J . Nutrient condutivity effects in sweet pepper using a nutrient film technique 2. Blossom-end rot and fruit mineral status. New Zealand J . Crop Hortic. Sci., 27:239-247, 1999.

THOMAS, J.R. \& HEILMAN, M.D. Influence of moisture and fertilizer on growth and $\mathrm{N}$ and $\mathrm{P}$ uptake by sweet peppers. Agron. J., 59:27-30, 1967.

VALENZUELA, J.L. \& ROMERO, L. Yield and optimum rate of nutrient in Capsicum annumm cv Lamuyo. Python Intern. J. Exp. Bot., 58:63-75, 1996.

VITTI, G.C. Avaliação e interpretação do enxofre no sol o e planta. J aboticabal, FUNEP, 1989. p.??

WOSTEN, J.H.M. \& van GENUCHTEN, M.TH. Using texture and other soil properties to predict the unsaturated soil hydraulic functions. Soil Sci. Soc. Am. J., 52:1762-1770, 1988. 